\title{
Some Effects of Multiple Scattering on Heating Rates in the Ozone Layer
}

\author{
Benjamin M. Herman and Douglas N. Yarger \\ The University of Arizona, Tucson
}

(Manuscript received 28 July 1965, in revised form 1 December 1965)

\begin{abstract}
The effects of multiple scattering on the heating rates in the ozone layer are investigated. Computations are performed for two wavelengths, one rather highly absorbing, $\lambda=3112 \AA$, and one rather weakly absorbing, $\lambda=3323 \AA$, and for three solar elevation angles. These results are compared with heating rates computed on the basis of a Beer's law type of exponential absorption, neglecting all scattering. It is shown that, at the weakly absorbing wavelength, and for small zenith angles, the effect of scattering is such as to increase the heating rate by about 40 per cent. At the more highly absorbing wavelength, scattering effects are small and may safely be neglected.
\end{abstract}

\section{Introduction}

In a recent paper by the authors (Herman and Yarger, 1965) a series of calculations of the emergent intensities and polarizations were presented for two wavelengths in the near ultraviolet. One of the wavelengths, $3323 \AA$, is rather weakly absorbed while the other, $3112 \AA$, is highly absorbed by the atmospheric ozone layer. These calculations were presented for a solar zenith angle of $84^{\circ}$, and were performed by a numerical integration of the transfer equation described in another paper (Herman and Browning, 1965). A very useful by-product of the numerical method is that the intensities are computed at all intermediate levels throughout the atmosphere. By numerically integrating these intensities over solid angle, the upward and downward flux densities at each level may readily be determined, from which the heating rates may be calculated.

The problem of atmospheric heating due to the absorption of solar ultraviolet radiation has attracted the attention of numerous workers. Some of the more recent investigators include Johnson (1953), Craig and Ohring (1958), Murgatroyd and Goody (1958), and Manabe and Möller (1961). They make the common assumption that scattering effects may be neglected, and therefore, base their absorption calculations on Beer's law methods. It is the purpose of this paper to investigate the validity of this assumption for a strongly and weakly absorbing wavelength pair in the Huggins band region where scattering effects are apt to be most significant.

\section{General procedure}

Details of the numerical methods have been presented elsewhere (Herman and Browning, 1965) and need not be repeated. $A$ check of the numerical scheme for the non-absorbing case was made using the tables of
Coulson et al. (1960) as a standard. For optical depths ${ }^{1}$ $\tau$, up to and including $\tau=1.0$, the maximum error in the emergent intensities was approximately 2 per cent, with the majority of values being considerably more accurate. Since smaller increments in the numerical integration were used in the present work than were used in the checking procedure, it is estimated that 2 per cent could be an upper limit to the possible error. All calculations were performed on the IBM 7072 computor of the Numerical Analysis Laboratory of the University of Arizona.

Calculations were performed for two wavelengths, $\lambda=3112 \AA$ and $\lambda=3323 \AA$, and for three solar zenith angles and one ozone distribution. Defining the cosine of the solar zenith angle as $\mu_{0}$, the calculations were performed for $\mu_{0}=0.10453, \mu_{0}=0.4$ and $\mu_{0}=0.9$. The experimental absorption coefficients were taken from the data of Inn and Tanaka (1959), which gave 0.122 $\mathrm{cm}^{-1}$ for $\lambda=3323 \AA$ and $2.464 \mathrm{~cm}^{-1}$ for $\lambda=3112 \AA$. The particular ozone distribution used was taken from a sounding at Fort Collins, Colorado, on 6 February 1963 , supplied by Mr. W. S. Hering. The total ozone amount for this sounding, as determined by a Dobson spectro-photometer was $0.269 \mathrm{~atm}-\mathrm{cm}$. Since this sounding terminated at about the $10-\mathrm{mb}$ level, a constant mixing ratio of $9 \mu \mathrm{g} \mathrm{gm}^{-1}$ was assumed above this level. This resulted in an integrated total ozone amount of $0.2686 \mathrm{~atm}-\mathrm{cm}$, determined by planimetering the corresponding area on an ozonagram. This distribution is shown in Fig. 1. The total optical depth $\tau_{T}$ for this sounding was 1.537 at $\lambda=3112 \AA$ and 0.69 for $\lambda=3323 \AA$.

As mentioned earlier, calculations of the upward and downward intensities at each level were performed. For

1 The optical depth at the height, $Z$, and at wavelength $\lambda$, is defined as $\tau_{\lambda}(Z)=\int_{z}^{\infty} k_{\lambda \tau} \rho d Z$, where $k_{\lambda_{r}}$ is the mass attenuation coefficient at wavelength $\lambda$, and $p$ is the density of the attenuating gas. 


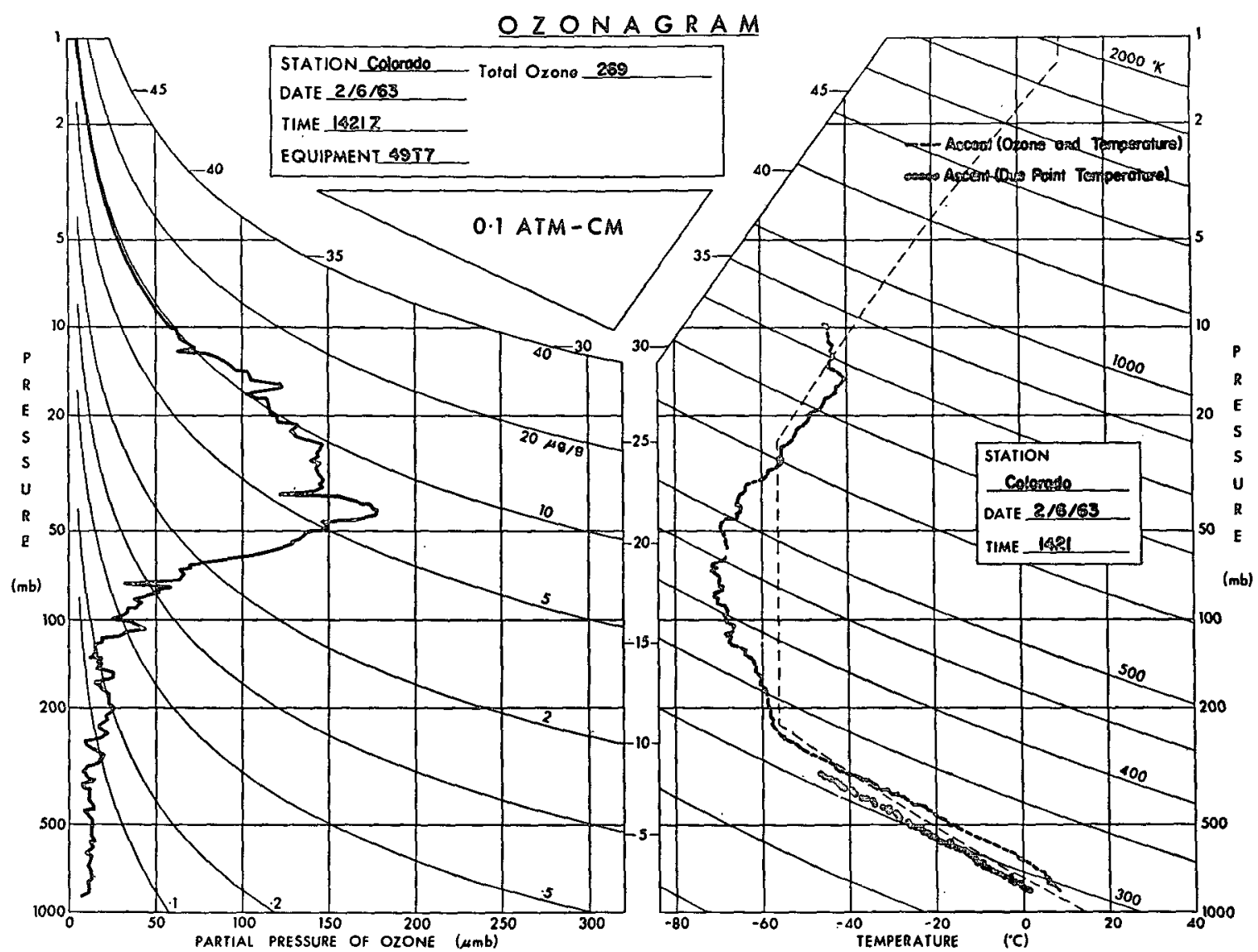

FIG. 1. Vertical distribution of ozone over Fort Collins, Colorado, on 6 February 1963.

the present calculations, increments at $\Delta \tau=0.005$ were used at $\lambda=3323 \AA$ and $\Delta \tau=0.01$ at $\lambda=3112 \AA$. These increments correspond to pressure increments of about $7 \mathrm{mb}$ near the surface (approximately 900-mb level for Fort Collins) to about $4 \mathrm{mb}$ at the $10-\mathrm{mb}$ level at $\lambda=3323 \AA$, and to about $11 \mathrm{mb}$ and $0.9 \mathrm{mb}$, respectively, at $\lambda=3112 \AA$. This increment of pressure is quite variable within the ozone layer because of the rapidly varying absorption optical depth ${ }^{2}$ due to the varying ozone concentration. At the level of maximum ozone $(\approx 40 \mathrm{mb})$ the absorption optical depth increment $\Delta \tau_{a}$ is about 80 per cent of the total increment of optical depth $\Delta \tau$, while near the ground the value becomes about 3 per cent at $\lambda=3112 \AA$.

The upward flux density at any level $F_{\lambda}(\tau)$ is related to the upward intensities at that level through the expression

$$
F_{\lambda}(\tau) \uparrow=\int_{\mu=0}^{\mu=1} \int_{\varphi=0}^{2 \pi} I_{\lambda}(\tau, \mu, \varphi) \mu d \mu d \varphi,
$$

where $\mu=\cos \theta$, and $I_{\lambda}(\tau, \mu, \varphi)$ is the intensity at the

2 The absorption optical depth is defined, analogously to the total optical depth, as $\tau_{\lambda a}(Z)=\int_{Z}^{\infty} k_{\lambda a} \rho_{a} d Z$, where $k_{\lambda a}$ is the mass absorption coefficient and $\rho_{a}$ is the density of absorbing gas, in this case ozone. level $\tau$ in the direction specified by $\mu, \varphi$. Similarly, the downward flux density is given as

$$
F_{\lambda}(\tau) \downarrow=\int_{\mu=-1}^{0} \int_{\varphi=0}^{2 \pi} I_{\lambda}(\tau, \mu, \varphi) \mu d \mu d \varphi+F_{s \lambda}(\tau),
$$

where $F_{s \lambda}(\tau)$ is the incident solar radiation at wavelength $\lambda$ and at the depth $\tau$, and is given by $F_{s \lambda}(0) e^{-\left(\tau / \mu_{0}\right)}$. The integrations in Eqs. (1) and (2) were performed numerically from the computed intensities.

The rate of energy absorbed per unit mass, $d h / d t$, in a horizontally homogeneous atmosphere, is given by

$$
\frac{d h}{d t}=g \frac{\partial F_{z n}}{\partial p}
$$

where $g$ is the gravitational constant, $p$ is pressure, and $F_{Z_{n}}$ is the net flux in the $Z$ direction, considered positive upwards. At the level $\tau$ and at wavelength $\lambda$, the net flux is given by

$$
F_{Z_{n \lambda}}(\tau)=F_{\lambda}(\tau) \uparrow+F_{\lambda}(\tau) \downarrow,
$$

where $F_{\lambda}(\tau) \uparrow$ and $F_{\lambda}(\tau) \downarrow$ are given by Eqs. (1) and (2). The procedure then was to calculate $F_{Z n \lambda}(\tau)$ at increments of $\Delta \tau=0.005$ for $\lambda=3323 \AA$ and $\Delta \tau=0.01$ 


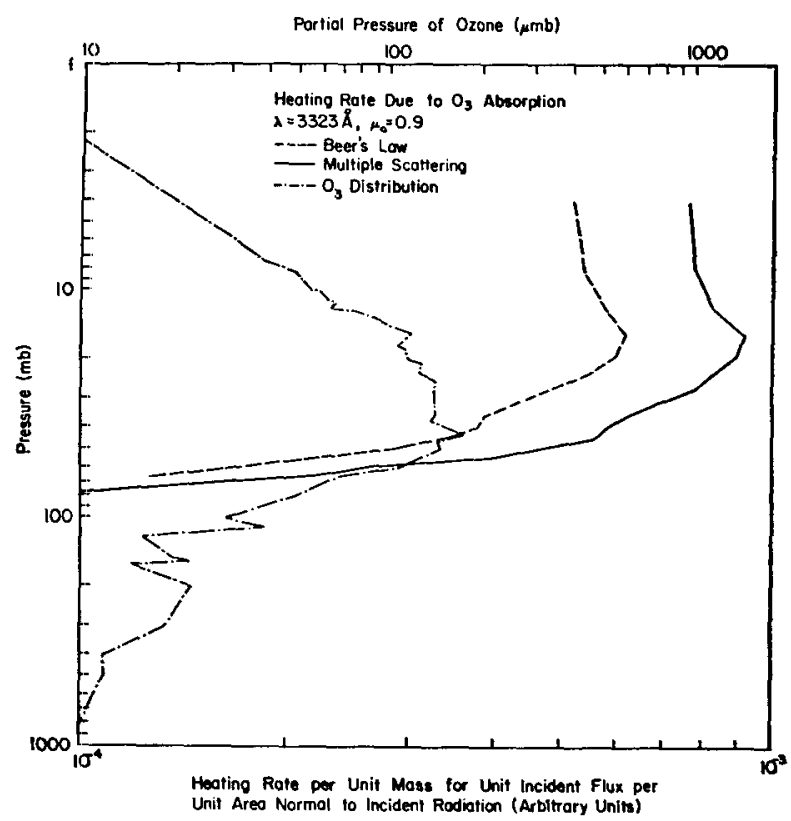

Fig. 2. Effect of multiple scattering on atmospheric heating for $\lambda=3323 \AA$ and $\mu_{0}=0.9$.

at $\lambda=3112 \AA$. The increment of $\Delta p$ corresponding to the $\Delta \tau$ increment at any level was determined from curves of $\tau$ vs. $p$, prepared in a manner described in an earlier paper (Herman and Yarger, 1965). From the value of $\Delta F_{Z n \lambda} / \Delta p$ thus obtained, the incremental heating rate $\Delta h / \Delta t$ could then be obtained from the finite difference approximation to Eq. (3). All calculations assumed incident plane parallel radiation of unit energy per unit area normal to the beam per unit time.

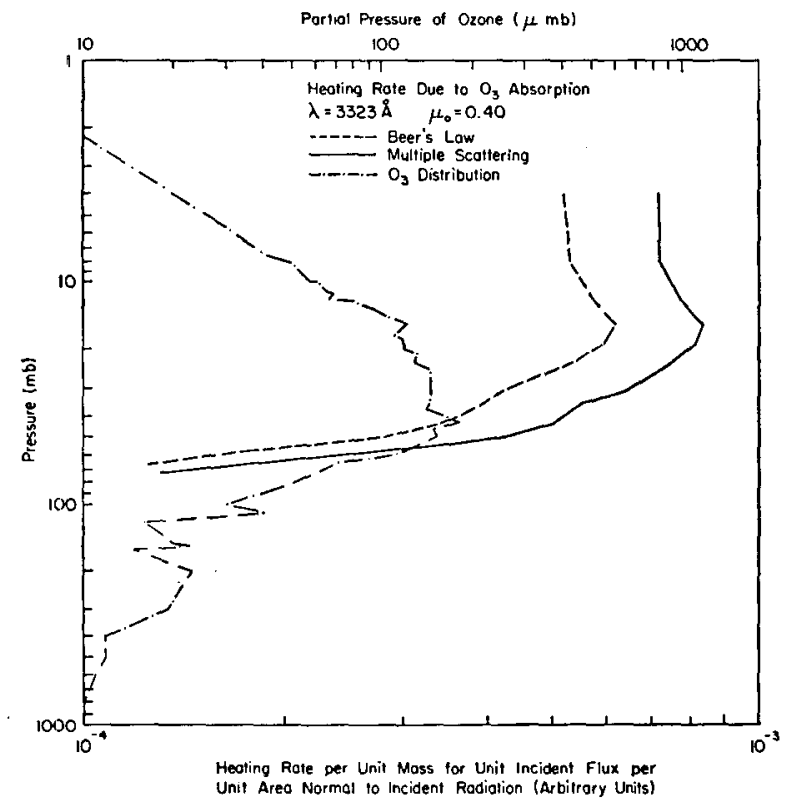

FIG. 3. Effect of multiple scattering on atmospheric heating for $\lambda=3323 \AA$ and $\mu_{0}=0.4$

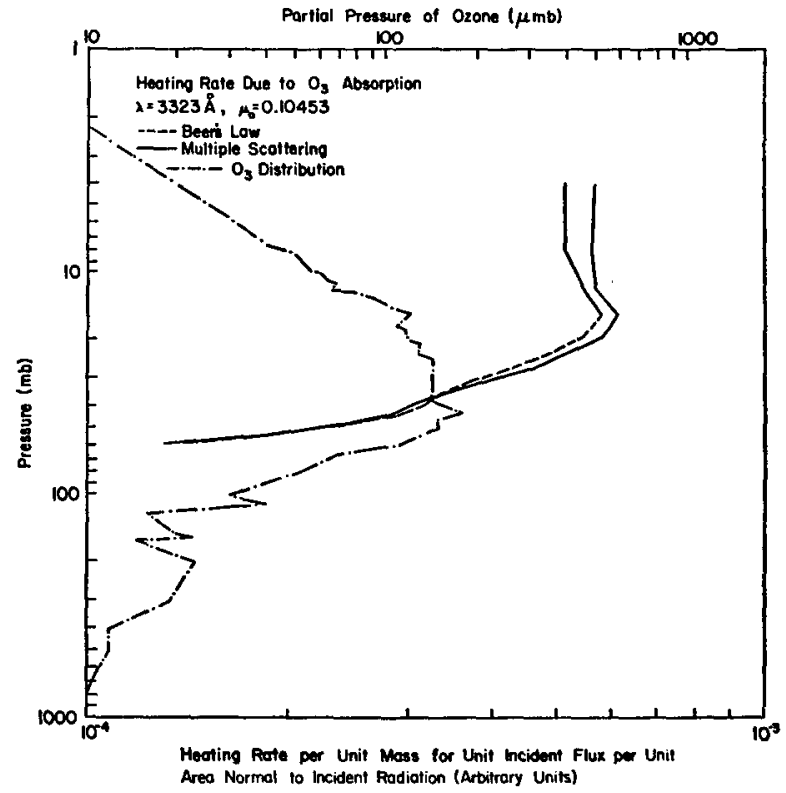

FIG. 4. Effect of multiple scattering on atmospheric heating for $\lambda=3323 \AA$ and $\mu_{0}=0.10453$.

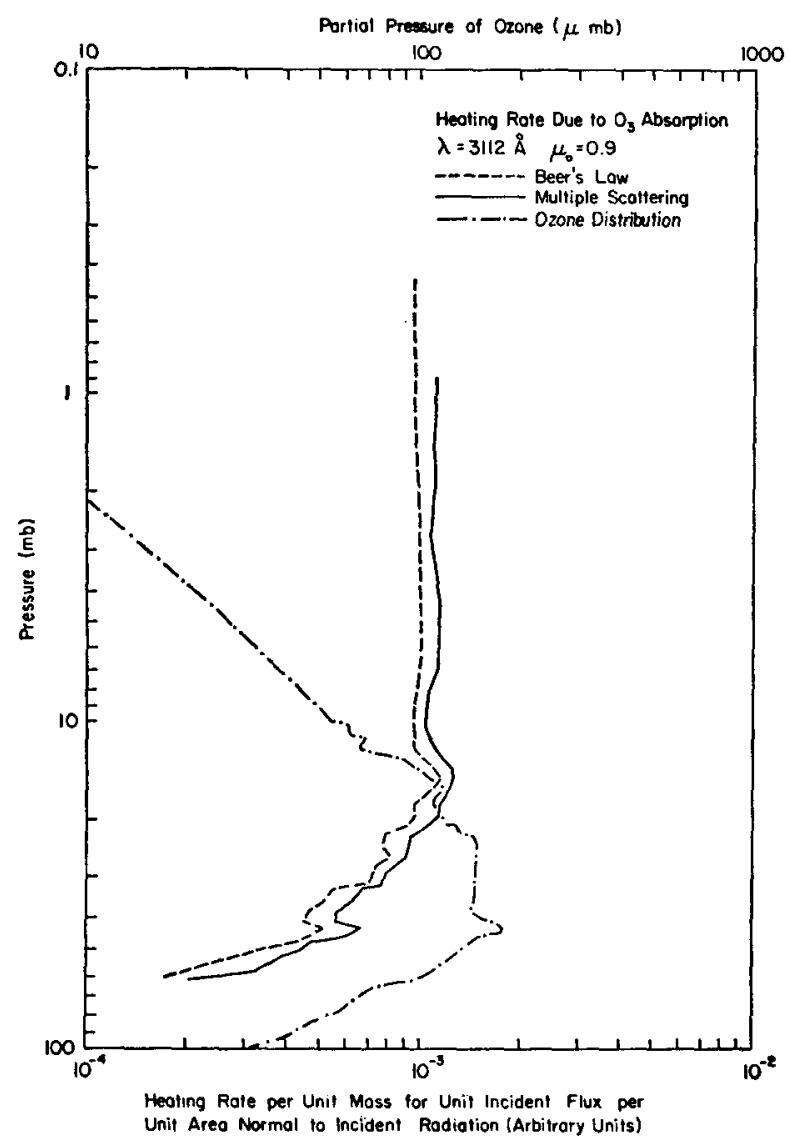

Fig. 5. Effect of multiple scattering on atmospheric heating for $\lambda=3112 \AA$ and $\mu_{0}=0.9$. 


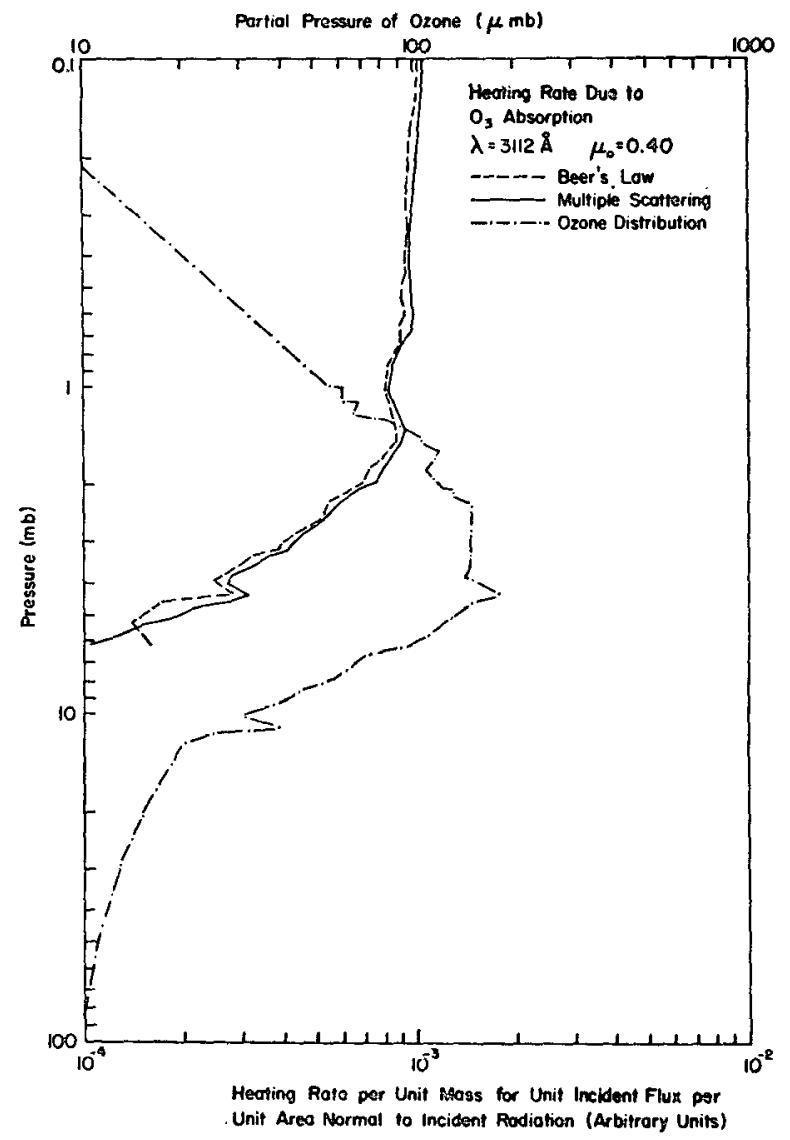

FIG. 6. Effect of multiple scattering on atmospheric heating for $\lambda=3112 \AA$ and $\mu_{0}=0.4$.

Curves of the heating rate for the same ozone distribution at the same two wavelengths and the same solar zenith angles were also prepared assuming only a Beer's law exponential absorption, neglecting all scattering. For this case, the downward solar radiation $F_{\circ \lambda}(\tau)$, at any level $\tau$, is given by

$$
F_{s \lambda}(\tau)=F_{s \lambda}(0) e^{-\left(\tau_{a} / \mu_{0}\right)} .
$$

It then follows that

$$
\frac{\Delta h}{\Delta t}=g \frac{\Delta F_{\mathrm{a} \lambda}(\tau)}{\Delta p}=g \frac{F_{\mathrm{s \lambda}}(\tau)\left[1-e^{-\left(\Delta \tau_{a} / \mu_{0}\right)}\right]}{\Delta p},
$$

where $\Delta \tau_{a}$ is the increment of absorption optical depth corresponding to the pressure increment $\Delta p$. Again, these values were determined from a curve of $\tau_{a}$ vs. $p$ prepared as described in the earlier paper (Herman and Yarger, 1965).

\section{Results and discussion}

Results of the heating rates, prepared from the two methods, are shown in Figs. 2-7. Examination of Figs. 2, 3 and 4 shows that at $\lambda=3323 \AA$ the effect of scattering on the heating rate due to absorption in-

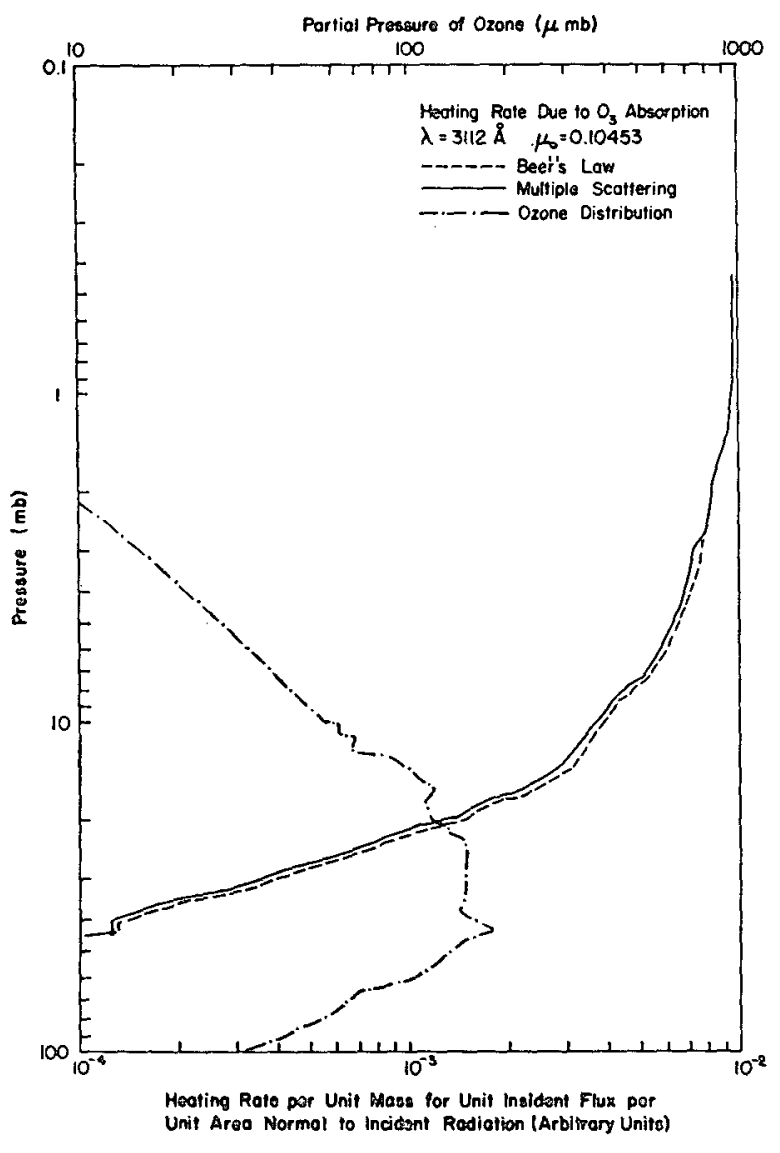

Fig. 7. Effect of multiple scattering on atmospheric heating for $\lambda=3112 \AA$ and $\mu_{0}=0.10453$.

creases as $\mu_{0}$ increases. At $\mu_{0}=0.9, p=15 \mathrm{mb}$ (Fig. 2), it is seen that the heating rate with scattering is about $9.4 \times 10^{-3}$ (arbitrary units) as opposed to the Beer's law result of $6.7 \times 10^{-3}$. This is an increase of about 40 per cent. At $\mu_{0}=0.4$ (Fig. 3), this figure is about 35 per cent while at $\mu_{0}=0.10453$ (Fig. 4), it is about 7 per cent. At the more highly absorbing wavelength $(\lambda=3112 \AA)$ the difference between the two methods is quite small at all $\mu_{0}$ (Figs. 5, 6, 7). However, it can be seen that at $\mu_{0}=0.9$ (Fig. 5), inclusion of scattering increases the absorption while at $\mu_{0}=0.10453$ (Fig. 7), scattering actually decreases the absorption. These effects may be explained in the following manner.

In the Beer's law calculations (no scattering) all radiation which is not absorbed is assumed to proceed in the direction of the incident beam, while in the computations allowing for scattering, some of the energy not absorbed is scattered into all directions according to the well-known Rayleigh scattering law's. The effect of the scattering is to cause the scattered beams to proceed through the absorbing layer in a zig-zag manner. The path length through the absorbing medium of the scattered beams will thus be different from that of the transmitted incident beam. For small solar zenith angles, the scattered beams will, on the average 
over all directions, have a longer path length than the incident beam, thus giving rise to considerably more absorption. As $\mu_{0}$ decreases with the sun approaching the horizon, the incident beam traverses a longer and longer path through the absorbing medium, and eventually a value of $\mu_{0}$ would be reached such that the effect of scattering is to shorten the effective path length in the absorbing medium. At $\lambda=3323 \AA$, this value of $\mu_{0}$ is obviously somewhat less than 0.10453 . The situation at $\lambda=3112 \AA$ is slightly different. At this highly absorbing wavelength, most of the energy can be accounted for by including only first order, or single scattering. For this situation, only one change in direction of a beam is of any consequence and, therefore, the average path of the scattered radiation will be less than for the case of weak absorption where many orders of scattering are important. Therefore, at a value of $\mu_{0}=0.10453$, the result of the scattering process is to decrease the path length at $\lambda=3112 \AA$ resulting in less absorption than for the Beer's law case.

One should note the fact that in no instances for the present examples does there appear to be a significant change in the level of maximum heating rate per unit mass. At $\lambda=3112 \AA$, however, the maximum is so broad that there exists the possibility that a finer increment of $\Delta \tau$ in the computations would reveal significant differences in the level of maximum heating.

Although the present study is by no means complete, it does point out the possibility of significant contributions to the absorption of ultraviolet radiation by the scattering process, particularly in the wing regions of the absorption bands. Although the overall absorption in these regions is less than in the central portions of the band, when integrated over all $\lambda$, it is likely that significant corrections to the heating rates would result, particularly for the larger values of $\mu_{0}$. Actual determination of these corrections will require calculations at several more values of $\mu_{0}$ and $\lambda$. Continuation of this work is planned for the future.

\section{REFERENCES}

Coulson, K. L., J. V. Dave and Z. Sekera, 1960: Tables Relaled to Radiation Emerging from a Planetary Atmosphere with Rayleigh Scattering. Berkeley and Los Angeles, University of California Press, 548 pp.

Craig, R. A., and G. Ohring, 1958: The temperature dependence of ozone radiational heating rates in the vicinity of the mesopeak. J. Meteor., 15, 59-62.

Herman, B. M., and S. R. Browning, 1965 : A numerical solution to the equation of radiative transfer. J. Atmos. Sci., 22, 559-566.

- , and D. N. Yarger, 1965: The effect of absorption on a Rayleigh atmosphere. J. Atmos. Sci., 22, 644-651.

Inn, E. C. Y., and Y. Tanaka, 1959: Ozone absorption coefficients in the visible and ultraviolet regions. Advances in Chemistry Series, No. 21, American Chemical Society, 263-268.

Johnson, F. S., 1953: High-altitude diurnal temperature changes due to ozone absorption. Bull. Amer. Meteor. Soc., 34, 106-110.

Manabe, S., and F. Möller, 1961: On the radiative equilibrium and heat balance of the atmosphere. Mon. Wea. Rev., 89, 503-532.

Murgatroyd, R. J., and R. M. Goody, 1958: Sources and sinks of radiative energy from 30 to $90 \mathrm{~km}$. Quart. J.R. Meteor. Soc., 84, 225-234. 